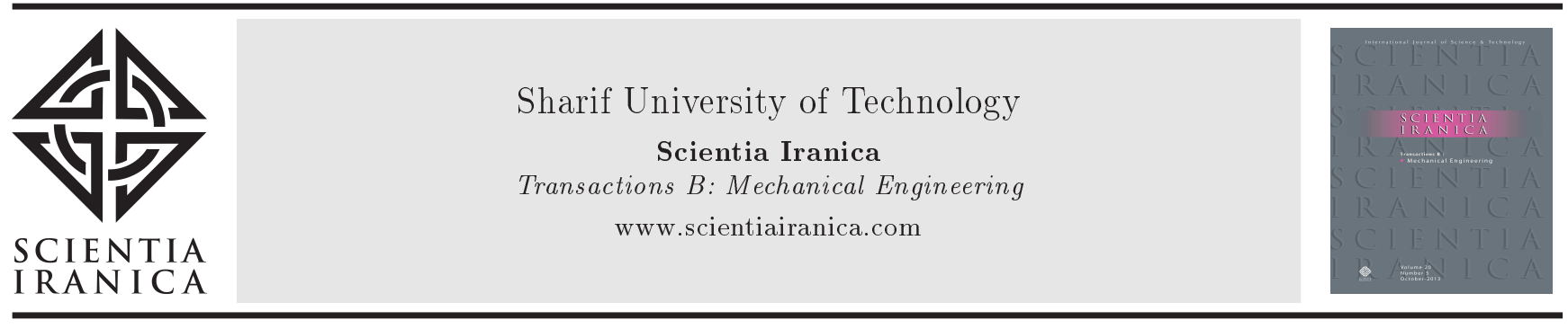

\title{
An investigation into the accelerometer mounting effects on signal transmissibility in modal measurements
}

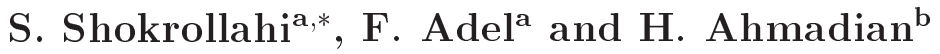 \\ a. Institute of Space Research, Laboratory of Modal Testing, Malek Ashtar University of Technology, Lavizan, Tehran, P.O. Box \\ $14665 / 143$, Iran. \\ b. School of Mechanical Engineering, Iran University of Science and Technology, Narmak, Tehran, P.O. Box 16844, Iran.
}

Received 10 February 2016; received in revised form 21 June 2016; accepted 8 October 2016

\section{KEYWORDS \\ Accelerometer; \\ Mounting dynamics; \\ Signal \\ transmissibility; \\ Modal testing.}

\begin{abstract}
The purpose of this study is to define a simple model and discuss the main effects of the use of the sensors with imperfect mounting in experimental measurements. This paper presents a theoretical and experimental investigation into the effects of different mounting methods of accelerometers on signal transmissibility in modal testing. In the theoretical part, a 2-Degree-Of Freedom (2-DOF) model is used, where the first DOF accounts for the accelerometer seismic mass and Piezo-crystal and the second DOF represents the mounting interface dynamics. An experimental modal analysis is conducted on a simple steel free-free beam using impact hammer excitation. The time domain signals and Frequency Response Functions (FRFs) are measured in the cases of magnet, wax, and stud mounting. It is found that the method of mounting has a significant effect on damping rates of measured responses. Although natural frequencies undergo no important changes, the quality of measured FRFs is degraded considerably.

(C) 2017 Sharif University of Technology. All rights reserved.
\end{abstract}

\section{Introduction}

The technique of attaching contact-type sensors (such as accelerometers) can have a significant effect on the sensed signal quality in an experimental measurement. When the accelerometers are not installed properly on the surface of the test structure, erroneous signals would be produced, which will significantly degrade the measurement accuracy [1-3]. In general, sensor mounting dynamics are ignored in modal testing based on the assumption that they have negligible effect on output response, compared to the other factors such as mass loading $[4,5]$. There is no known reason for

\footnotetext{
*. Corresponding author. Tel.: +9821 22285399;

Fax: +982122987004

E-mail addresses: s_shokrollahi@mut.ac.ir (S. Shokrollahi);

fad@mut.ac.ir (F.Adel); ahmadian@iust.ac.ir (H.

Ahmadian)
}

doi: $10.24200 /$ sci.2017.4244 this opinion. Moreover, if the motion of the structure is not correctly transmitted to the sensor, due to poor mating conditions, it cannot be truly measured and may cause inaccurate data. Two important mounting parameters, which affect the signal transmissibility and FRF quality, are shown in the simplified diagram in Figure 1. As shown in Figure 1(b), the nonrigid coupling of the accelerometer to the structure can cause a measured acceleration that cannot be a representative of the structure [6]. In Figure 1(b), $A$ is the accelerometer; $M$ is a means of mounting; $a_{s}$ is the acceleration of the structure; and $a_{A}$ is the acceleration sensed by the accelerometer.

According to the military and civil standards $[7,8]$, method of mounting and condition of mounting surface should be stated in any report. There are several different mounting methods available such as stud, wax, magnet, etc. [8]. The reliability of the measured results from modal testing depends not only on the basic features of the accelerometer (i.e., 


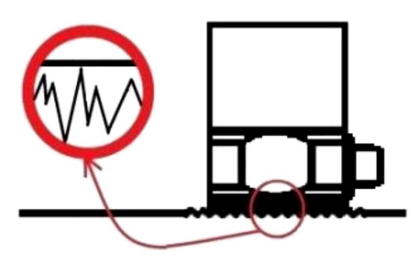

(a)

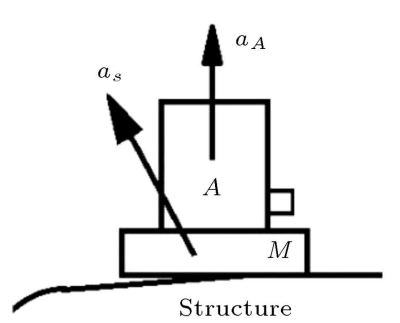

(b)
Figure 1. Two important parameters affecting transmissibility: (a) Surface irregularities and (b) poor mounting.

type, manufacturer, etc.), but also on the way it is attached to the test structure. The recommended mounting method depends upon the dynamic measurement requirements, such as frequency range, mounting location, prohibitions, accessibility, and temperature.

It is widely known [9] that the accelerometer attachment techniques have a significant effect on its mounted resonance and frequency response characteristics. Bowers et al. [10] studied the effectiveness of different mounting methods on the mounted resonance of an accelerometer and accuracy and repeatability of data. Peres et al. [11] overviewed some of the common problems related to mounting and dynamic coupling between shaker, stinger, and test object that are typically encountered in experimental measurements. Colombo et al. [12] investigated the effects of different couplant materials on measured frequency responses and proposed the "plasticine" material for attaching accelerometers to the surface of the structures.

The importance of accelerometer modeling for calibration purposes has been discussed in few studies [13,14]. Link et al. [15] proposed a model comprised of a linear, second-order differential equation with unknown coefficients. Täubner et al. [16] showed that the coupled mechanical system of the accelerometer with the armature could be described as a two-mass oscillator, one mass being the seismic mass and the second mass being the sum of the armature and the transducer base. Bruns et al. [17] performed more detailed measurements integrated with 2-DOF modeling for accelerometer and its mounting, which led to a better understanding of what happened in [16].

Although accelerometers have had widespread use in vibration sensing purposes, one aspect of their application has drawn minor attention, namely, the effect of the mounting dynamics on the measured structural response [18]. To the best of the authors' knowledge, the dependence of the vibration transmissibility and FRF quality on the accelerometer mounting method has not yet been studied. This study investigates the effects of mounting dynamics on the response of a typical structure in the time and frequency domains, separately. Since different mounting techniques may affect transmissibility, it is necessary to understand well the mechanics of mounting. The mechanics of a nonrigid mount and their effect on signal transmission and response amplification should be examined.

\section{The mechanics of mounting}

A simplified 2-DOF model is shown in Figure 2(a), which is considered for studying some important effects of neglecting interface dynamics.

As it can be seen from Figure 2, the accelerometer and its mounting interface are modeled separately as 1-DOF systems. The accelerometer parameters are seismic mass, $m_{s}$; piezo-crystal stiffness, $k_{s}$; and piezo-crystal damping coefficient, $c_{s}$. When mounting dynamics are included, the model appears as shown in Figure 2(a). Considering the possibility of relative motion between the test structure and the base of the accelerometer, the mounting interface having additional elastic properties could also be modeled by a spring of stiffness, $k_{m}$, and damping coefficient, $c_{m}$. The accelerometer base mass is denoted by $m_{b}$. When the mounting dynamics are neglected, the 1DOF model shown in Figure 2(b) will be obtained.
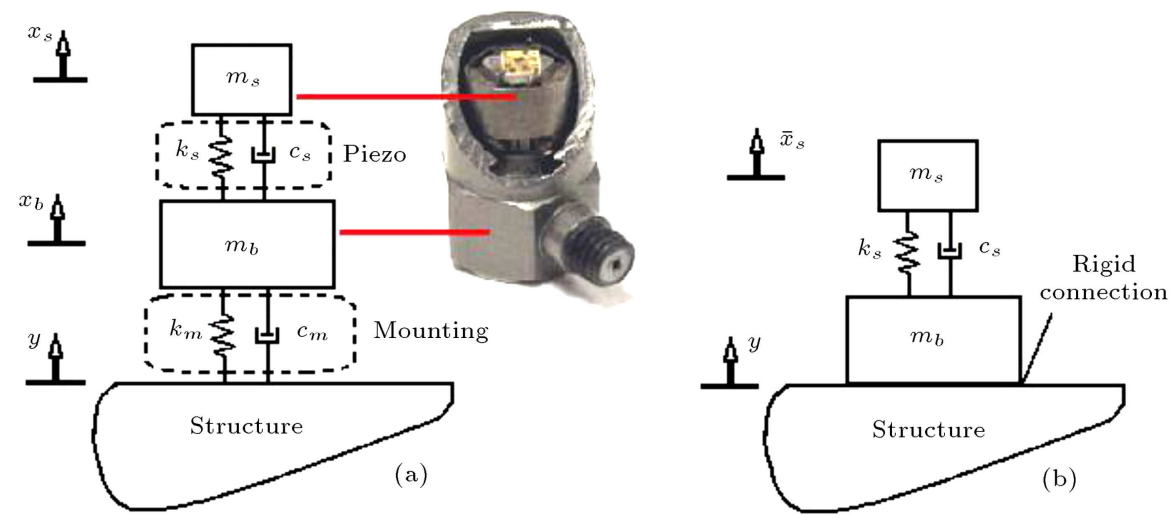

Figure 2. A simplified model to study the effects of mounting: (a) With mounting dynamics and (b) without mounting dynamics. 
Note that, in the latter case, the excitation motion, $y(t)$, is directly applied to the accelerometer whereas, in the former case, it is applied through the interface. In Figure 2(a), a cutaway of a typical accelerometer is shown for the convenience of the reader.

Transmission of signals from test object onto the accelerometer is good only when the mounting interface allows to. If the motion of the structure is taken as the forced oscillation, $y=y_{0} \sin \omega t$, then $m_{b}$ and $m_{s}$ can go vibrated in the general form of $x_{b}=x_{b 0} \sin (\omega t-\alpha)$ and $x_{s}=x_{s 0} \sin (\omega t-\beta)$, respectively. Now, the equations of motion can be described by the differential equation system:

$$
\begin{aligned}
& m_{s} \ddot{x}_{s}+c_{s}\left(\dot{x}_{s}-\dot{x}_{b}\right)+k_{s}\left(x_{s}-x_{b}\right)=0, \\
& m_{b} \ddot{x}_{b}+c_{m}\left(\dot{x}_{b}-\dot{y}\right)+k_{m}\left(x_{b}-y\right)-c_{s}\left(\dot{x}_{s}-\dot{x}_{b}\right) \\
& \quad-k_{s}\left(x_{s}-x_{b}\right)=0 .
\end{aligned}
$$

By defining the operator $D=\frac{d}{d t}$, Eqs. (1) and (2) can be rewritten as:

$$
\begin{aligned}
& \left(m_{s} D^{2}+c_{s} D+k_{s}\right) x_{s}=\left(c_{s} D+k_{s}\right) x_{b}, \\
& {\left[m_{b} D^{2}+\left(c_{m}+c_{s}\right) D+k_{m}+k_{s}\right] x_{b}} \\
& \quad=\left(c_{s} D+k_{s}\right) x_{s}+\left(c_{m} D+k_{m}\right) y .
\end{aligned}
$$

Converting Eqs. (3) and (4) into the frequency domain, $(D \equiv i \omega)$ yields:

$$
\begin{aligned}
& \left(-m_{s} \omega^{2}+i c_{s} \omega+k_{s}\right) x_{s}(\omega)=\left(i c_{s} \omega+k_{s}\right) x_{b}(\omega) \\
& {\left[-m_{b} \omega^{2}+i\left(c_{m}+c_{s}\right) \omega+k_{m}+k_{s}\right] x_{b}(\omega)} \\
& \quad=\left(i c_{s} \omega+k_{s}\right) x_{s}(\omega)+\left(i c_{m} \omega+k_{m}\right) y(\omega) .
\end{aligned}
$$

By combining Eqs. (5) and (6), the system frequency transfer function is expressed by Eq. (7) as shown in Box I.

For analysis, damping of piezoelectric crystal, $c_{s}$, is neglected in this model, because accelerometers have very low damping factors [19]. On the other hand, in basic calculations of piezoelectric effect, it is not usual to consider damping ratios [20]. Also, by assuming mounting damping, $c_{m}$, close to zero, it is concluded that:

$$
\frac{x_{s}(\omega)}{y(\omega)}=\left[\frac{k_{m} k_{s}}{\left(k_{s}-m_{s} \omega^{2}\right)\left(k_{s}+k_{m}-m_{b} \omega^{2}\right)-\left(k_{s}\right)^{2}}\right]
$$

The absolute displacement transmissibility, $T_{A}^{D}$, is defined as:

$$
T_{A}^{D}=\frac{x_{s 0}(\omega)}{y_{0}(\omega)}=\frac{k_{m} k_{s}}{\left(k_{s}-m_{s} \omega^{2}\right)\left(k_{s}+k_{m}-m_{b} \omega^{2}\right)-\left(k_{s}\right)^{2}}
$$

By defining $\omega_{s}=\sqrt{\frac{k_{s}}{m_{s}}}, \omega_{m}=\sqrt{\frac{k_{m}}{m_{b}}}$, and $r=\frac{k_{s}}{k_{m}}$, the dimensionless form of Eq. (9) is obtained:

$$
T_{A}^{D}=\frac{x_{s 0}(\omega)}{y_{0}(\omega)}=\frac{1}{\left[1-\left(\frac{\omega}{\omega_{s}}\right)^{2}\right]\left[1-\left(\frac{\omega}{\omega_{m}}\right)^{2}+r\right]-r}
$$

The relationship between the transmissibility, $T_{A}^{D}$, excitation frequency, $\omega$, accelerometer resonance frequency, $\omega_{s}$, mounting resonance frequency, $\omega_{m}$, and stiffness ratio, $r$, is obvious in Eq. (10). $\omega_{s}$ is the resonance frequency of the accelerometer measured in free space and it would be different if mounted on a structure. The mounted resonant frequency, $\omega_{m}$, of this system will definitely be lower than that of the accelerometer alone. The parameter $r$ is the stiffness ratio of piezoelectric crystal to the mounting interface.

In rigid mounting condition (Figure 2(b)), the principle of operation of an accelerometer can be explained by a seismic mass, $m_{s}$, attached to a piezocrystal with stiffness $k_{s}$ and damping $c_{s}$ that is, in turn, attached to the structure. Therefore, Eq. (7) is simplified to the following frequency transfer function:

$$
\frac{\bar{x}_{s}(\omega)}{y(\omega)}=\frac{\left(i c_{s} \omega+k_{s}\right)}{\left(-m_{s} \omega^{2}+i c_{s} \omega+k_{s}\right)} .
$$

Again, by considering zero value for the damping of piezoelectric crystal, $c_{s}$, the absolute displacement transmissibility in this case takes the form:

$$
\bar{T}_{A}^{D}=\frac{\bar{x}_{s 0}(\omega)}{y_{0}(\omega)}=\frac{1}{1-\left(\frac{\omega}{\omega_{s}}\right)^{2}} .
$$

One may consider that the displacement transmissibility is achieved in Eqs. (10) and (12) while the measured responses refer to the acceleration. This poses no problem as acceleration and displacement are only two different forms of presenting the same response. Multiplying the numerator and denominator of Eqs. (10) and (12) by $-\omega^{2}$ yields the acceleration transmissibility, which has no difference with displacement transmissibility.

$$
\frac{x_{s}(\omega)}{y(\omega)}=\left[\frac{\left(i c_{m} \omega+k_{m}\right)\left(i c_{s} \omega+k_{s}\right)}{\left(-m_{s} \omega^{2}+i c_{s} \omega+k_{s}\right)\left[-m_{b} \omega^{2}+i\left(c_{m}+c_{s}\right) \omega+k_{s}+k_{m}\right]-\left(i c_{s} \omega+k_{s}\right)^{2}}\right] .
$$


To determine the response amplification, $R A$, in different mounting conditions, the ratio $x_{s 0} / \bar{x}_{s 0}$ represents the accelerometer response amplification when mounting dynamic effects are considered for a harmonic excitation:

$$
R A=\frac{x_{s 0}(\omega)}{\bar{x}_{s 0}(\omega)}=\frac{1-\left(\frac{\omega}{\omega_{s}}\right)^{2}}{\left[1-\left(\frac{\omega}{\omega_{s}}\right)^{2}\right]\left[1-\left(\frac{\omega}{\omega_{m}}\right)^{2}+r\right]-r} .
$$

Using appropriate values for $r$ and $\omega_{m}$ in different mounting conditions can yield different transmissibility values. $\omega_{s}$ is the same for all mounting conditions. The process of choosing suitable values for $\omega_{m}, \omega_{s}$, and $r$ is argued in Appendix. Assuming $\omega_{s}$ as a constant quantity, it can be possible to do parametric study on $r, \omega_{m}$, and $\omega$ using MATLAB software. Figure 3 shows the absolute displacement transmissibility due to four different mounting conditions corresponding to Eqs. (10) and (12) up to $f_{\max }=3 \mathrm{kHz}$ for the parameter values given in Table 1 . Choosing $f_{\max }=$ $3 \mathrm{kHz}$ in numerical simulation is not a special selection and it could be selected of frequencies higher than $3 \mathrm{kHz}$. Interpretation of the results could be easier when the values of the response ratios are compared for various mounting conditions at different frequencies. In rigid condition, the mount responds in a way that the accelerometer response almost entirely reproduces

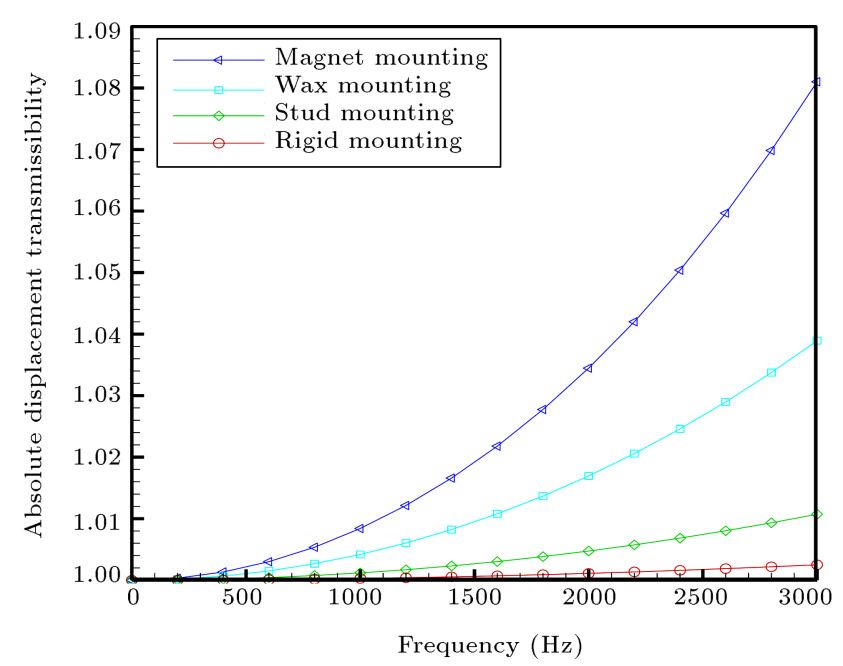

Figure 3. Transmissibility in four different mounting conditions. the input acceleration. Under the other conditions, the mount distorts the response, which may in fact make the recorded data worthless.

Figure 4 shows three curves of response amplification for stud, wax, and magnet mounting methods, corresponding to Eq. (13). Amplification of the response for the three different mounting conditions, which is derived from Figure 4, is given in Table 1 at two different frequencies of $\omega_{1}=2 \pi \times 1000 \mathrm{rad} / \mathrm{sec}$ and $\omega_{2}=2 \pi \times 3000 \mathrm{rad} / \mathrm{sec}$. This amplification, which is obvious in magnet mounting, is attributed to the relative motion among test structure, base mass, and seismic mass. In these mounting conditions, the accelerometer response is a compound of test structure and accelerometer base vibrations.

As it is evident from Figure 4, the stud mounting has the minimum amplification of the response. This means that, according to Eq. (13), the structural response has the minimum deviation in stud mounting from a rigid mounting. The wax and magnet mounting methods are in the subsequent places. The stud mounting method also has the minimum signal transmission (Figure 3), which means, in turn, the input excitation amplitude is transmitted to the accelerometer with a minimum variation. In other words, minimum relative motion exists between seismic mass and test structure according to Eq. (10). The wax and magnet mounting methods are again in next places.

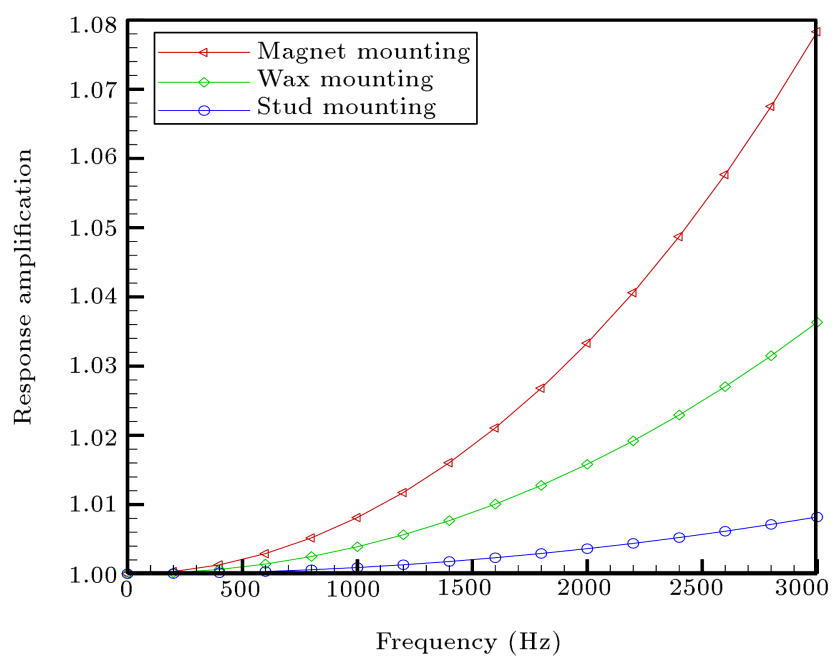

Figure 4. Response amplification in three non-rigid mounting conditions.

Table 1. Estimation of simulation parameters for the second-order model.

\begin{tabular}{cccccc}
\hline $\begin{array}{c}\text { Mounting } \\
\text { method }\end{array}$ & $\boldsymbol{\omega}_{\boldsymbol{s}}\left(\frac{\mathrm{rad}}{\mathrm{sec}}\right)$ & $\boldsymbol{\omega}_{\boldsymbol{m}}\left(\frac{\mathrm{rad}}{\mathbf{s e c}}\right)$ & $\boldsymbol{r}$ & $\begin{array}{c}\text { Amplification at } \\
\boldsymbol{\omega}_{\mathbf{1}}=\mathbf{2} \boldsymbol{\pi} \times \mathbf{1 0 0 0}\left(\frac{\mathbf{r a d}}{\mathbf{s e c}}\right)\end{array}$ & $\begin{array}{c}\text { Amplification at } \\
\boldsymbol{\omega}_{\mathbf{2}}=\mathbf{2} \boldsymbol{\pi} \times \mathbf{3 0 0 0}\left(\frac{\mathrm{rad}}{\mathbf{s e c}}\right)\end{array}$ \\
\hline Rigid & $2 \pi \times 60000$ & - & - & 1 & 1 \\
Stud & $2 \pi \times 60000$ & $2 \pi \times 40000$ & 1 & 1.001 & 1.008 \\
Wax & $2 \pi \times 60000$ & $2 \pi \times 30000$ & 10 & 1.004 & 1.036 \\
Magnet & $2 \pi \times 60000$ & $2 \pi \times 20000$ & 20 & 1.008 & 1.078 \\
\hline
\end{tabular}


It is clear from theoretical analysis that the method of mounting has significant effects on vibration transmissibility and signal amplification, especially at higher frequencies. The ability of coupling the motion depends highly upon the method of mounting. In other words, the quality of the attachment affects the nature of the transmissibility and response amplification. The analogy on this section can be extended to include other situations where mating surfaces are prevented from ideal contact, such as when foreign particles are entrapped between mating surfaces or even when other types of surface irregularities exist. All of these poor mounting conditions can degrade the measured response of the accelerometer and make the FRF of the test structure deviate as shown in the next sections.

\section{Experiment}

Figure 5(a) shows the test set-up for measuring the dynamic response of the steel beam. The suspended free-free beam of dimensions $500 \times 40 \times 12 \mathrm{~mm}^{3}$ and mass of $1.88 \mathrm{~kg}$ is excited by means of an impact hammer. A SKIL(B) model LC-01A impulse hammer with nylon tip is used to generate the impacts. It is equipped with force sensor model CL-YD-303 with measuring range up to $2000 \mathrm{~N}$ to measure the excitation force. Six impacts are generated for each case to have a reliable sample of signals. As shown in Figure 5(b) and (c), YE6268-36 from SINOCERA Piezotronics Inc. was used as dynamic data acquisition system to simultaneously record the force and acceleration using YE7600 software. Signal processing has been performed by using $N$-modal software from SINOCERA.

The accelerometers are SINOCERA Piezotronics Inc. type CA-YD-1181, which have high sensitivity, low transverse sensitivity, and a broadband frequency range of 1-10000 Hz. They weigh about $10 \mathrm{~g}$ and are connected through coaxial cables. These IEPE, small-size accelerometers are mounted symmetrically on both ends and midpoint of the beam using three basic mounting methods, namely, wax, magnet, and stud, as shown in Figure 6. It is a common practice to use superglue for most applications. However, superglue is not implemented in this research due to some difficulties encountered in removing and cleaning the sensors. The sampling rate was taken to be $2 \mathrm{kHz}$ and the duration of recorded signals was 2.5 sec.

\section{Results}

In this section, the quality of measurements related to the mounting effects of the accelerometers are assessed in terms of structural response in the time and frequency domains and natural frequencies.


Figure 5. (a) Free-free beam. (b) Modal lab environment. (c) YE6268-36 dynamic data acquisition system.


Figure 6. Mounting methods: (a) Wax, (b) magnet, (c) and (d) stud. 


\subsection{Structural response in the time domain}

Structural responses from time domain measured by accelerometers are presented in Figures 7 to 9 . The prominent aspect in these figures is the presence of different response damping rates in three mounting situations.

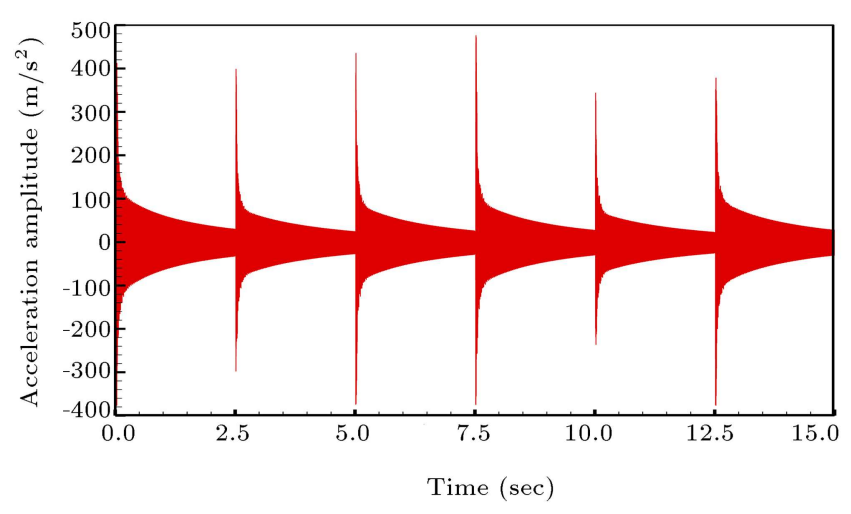

Figure 7. Measured time response (magnet mounting).



Figure 8. Measured time response (wax mounting).

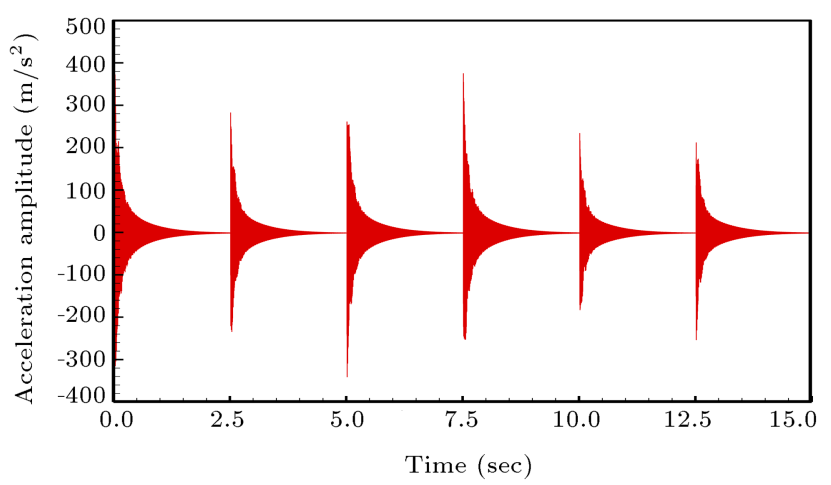

Figure 9. Measured time response (stud mounting).

\subsection{Structural response in the frequency domain}

Modal Indication Function (MIF) for the three cases is shown in Figure 10. From a practical point of view, comparison of the measured FRFs is a suitable way to assess the quality of the different mounting methods. If the mounting is not considered carefully, the measured response may be severely distorted as shown in Figure 10(a) and (b). In extreme cases, the recordings may be even worthless. Low noise and increasing quality of the MIF graph are clearly visible for stud mounting in Figure 10(c). Figure 11 shows all the measured frequency responses for a better comparison.

\subsection{Natural frequencies}

Table 2 represents the results for the first and second natural frequencies of the steel beam obtained from the Finite Element Method (FEM) and experimental measurements. It is found that the method of mounting has no significant effect on modes of vibration and there are no considerable changes in natural frequencies.

\section{Discussion}

The primary objective of this research is to investigate the effects of the accelerometer mounting dynamics on signal transmissibility and quality of the experimental measurements. For this purpose, a simple lumped mass model is developed for numerical simulations of signal transmission and response amplification for three mounting methods. Then, the experimental modal analysis is performed to observe mounting effects on the measured response quality. The obtained results from two approaches are in good conformity with each other in such a way that the stud mounting, for example, has the minimum amplification of response (Figure 4). This means that, according to Eq. (13), the structural response has the minimum deviation in stud mounting from a rigid mounting. The wax and magnet mounting methods are in the subsequent places. The stud mounting method also has the minimum signal transmission (Figure 3), which means, in turn, the input excitation amplitude is transmitted to the accelerometer with a minimum variation. In other words, minimum relative motion exists between the seismic mass and test structure according to Eq. (10). The wax and magnet mounting methods are again in next places.

Table 2. Natural frequencies in different mounting methods.

\begin{tabular}{cccccccc}
\hline \multirow{2}{*}{ Mode\# } & \multicolumn{7}{c}{ Natural frequency $(\mathbf{H z})$} \\
\cline { 2 - 8 } & FEM & Magnet & \%Diff & Wax & \%Diff & Stud & \%dDff \\
\hline 1 & 243.54 & 246.02 & 1.00 & 247.80 & 1.72 & 246.75 & 1.30 \\
2 & 670.90 & 674.54 & 0.54 & 665.77 & -0.77 & 669.95 & -0.14 \\
\hline
\end{tabular}




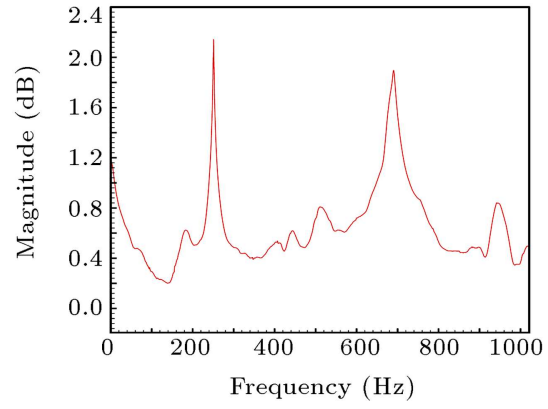

(a)

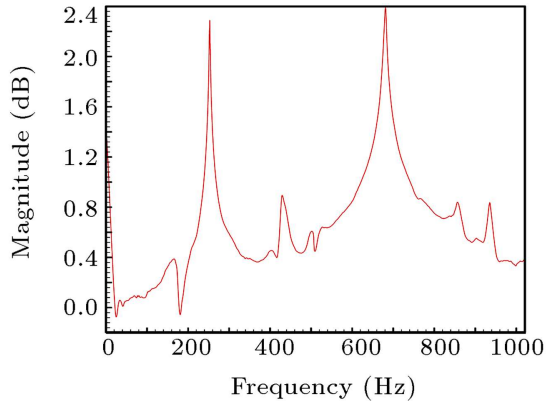

(b)

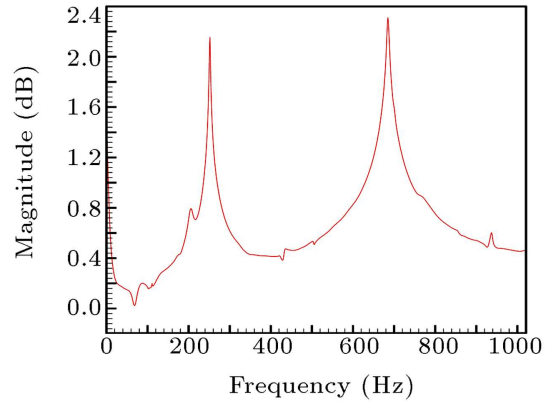

(c)

Figure 10. Measured frequency responses: (a) Magnet, (b) wax, and (c) stud.

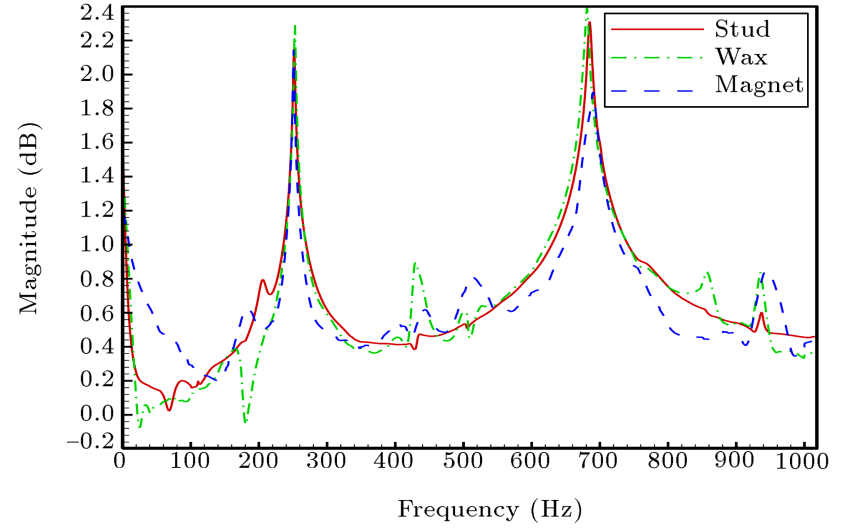

Figure 11. Measured frequency responses.

On the other hand, experimentally measured data are in good agreement with simulation results. Non-ideal mounting condition decreases the mounting stiffness and increases the response amplification and relative motion, causing time responses to decay slowly. It can be seen from Figures 7 to 9 that the highest damping rate belongs to the stud mounting. The stud mounting is much stiffer than the two other mounting methods. Thus, it undergoes slight relative motion and the response damps down rapidly (Figure 9). These small relative motions lead to a lower distortion and noise in frequency response in the stud mounting case as well (Figure 10(c)). But, in other cases, especially in the magnet mounting, the damping rate is lower due to the higher response amplification. The origin of the deviation in Figure 10(a) and (b) is the relative motion between the structure and the accelerometer. Thus, the frequency responses show higher deviations when the mounting rigidity decreases, and choosing the optimum mounting method will considerably improve the accuracy.

As it is evident from Figure 11, the preferred method for attaching an accelerometer is the stud mounting. This method yields the best results, because the accelerometer and test surface are essentially fused together by clamping force of the elastic stud, certifying accurate duplication of the motion of both bodies. The mounting wax is very comfortable to use; however, the more wax between surfaces, the greater will be the degradation of transmissibility. Magnetic mounting adapters are used to connect accelerometers to ferromagnetic surfaces such as machinery and structures where the instrument is to be commuted easily from point to point. Most magnetic adaptors are massive and they are useful only for low-frequency measurements.

\section{Conclusion}

In experimental modal analysis, it is usually assumed that the mounting condition, which is supposed to be rigid, faithfully transmits the response of the structure to the accelerometers. However, the current results show that this assumption is not valid for all mounting situations. The numerical simulation predicts that the mounting interface changes the signal transmissibility as well as amplifying the response, and this phenomenon introduces errors into the measured FRFs as seen in the experimental section. The experimental results show that the damping rates of output signals and quality of FRFs depend on the way the accelerometer is attached to the test structure, and neglecting the mounting dynamics can lead to a deviated and undesirable measured structural response in the time and frequency domains. To achieve the best measurement conditions in modal testing, it is important that the mounting surface of the accelerometers should be tightly coupled with the test surface, especially at higher frequencies. The model used in this paper is admittedly a rather simple one; but, it is applicable to a certain class of sensors and their mounting conditions. Theoretical modeling and parametric study combined with experimentally measured data have led to better understanding of mounting problems.

\section{References}

1. Tomczyk, K. and Layer, E. "Accelerometer errors in 
the measurement of dynamic signals", Measurement, 60, pp. 292-298 (2015).

2. Yanqiu, H., Juan, C. and Li Peng, Y. "Experimental analysis of accelerometer installation error", IEEE International Conference on Computer, Mechatronics, Control and Electronic Engineering, CMCE2010, China, pp. 40-42 (2010).

3. Tomczyk, K. "Impact of uncertainties in accelerometer modeling on the maximum values of absolute dynamic error", Measurement, 80, pp. 71-78 (2016).

4. Bi, S., Ren, J., Wang, W. and Zong, G. "Elimination of transducer mass loading effects in shaker modal testing", Mechanical Systems and Signal Processing, 38(2), pp. 265-275 (2013).

5. Karle, A.D., Bhoite, S.K. and Amale, A.B. "An analysis of transducer mass loading effect in shaker testing", International Journal of Engineering Research and Applications, 4(6), pp. 207-212 (2014).

6. Walter, P.L. "Accelerometer selection for and application to modal testing", 17th International Modal Analysis Conference, IMAC-XVII, USA, pp. 566-572 (1999).

7. Anon "Structureborne vibratory acceleration measurements and acceptance criteria of shipboard equipment", MIL-STD-740-2 (1986).

8. Anon "Mechanical vibration and shock - Mechanical mounting of accelerometers", ISO-5348 (1998).

9. Ewins, D.J., Modal Testing: Theory, Practice and Application, Second Edn., Research Studies Press, England (2000).

10. Bowers, S.V., Piety, K.R. and Piety, R.W. "Real-world mounting of accelerometers for machinery monitoring", Sound \& Vibration magazine, 25(2), pp. 14-23 (1991).

11. Peres, M.A., Bono, R.W. and Avitabile, P. "Effects of shaker, stinger and transducer mounting on measured frequency response functions", International Conference on Noise and Vibration Engineering, ISMA2012, Belgium, pp. 1475-1489 (2012).

12. Colombo, S., Giannopoulos, A., Forde, M.C., Hasson, R. and Mulholland, J. "Frequency response of different couplant materials for mounting transducers", NDT\&E International, 38(3), pp. 187-193 (2005).

13. Ripper, G.P., Dias, R.S. and Garcia, G.A. "Primary accelerometer calibration problems due to vibration exciters", Measurement, 42, pp. 1363-1369 (2009).

14. Hirunyapruk, C., Rattanangkul, P., Thummawut, B. and Plangsangmas, V. "Experimental investigation into the effects of exciter motions on the primary calibration of single-ended accelerometer", Measurement, 45, pp. 2407-2412 (2012).

15. Link, A., Täubner, A., Wabinski, W., Bruns, T. and Elster, C. "Modelling accelerometers for transient signals using calibration measurements upon sinusoidal excitation", Measurement, 40, pp. 928-935 (2007).
16. Täubner, A., Schlaak, H., Brucke, M. and Bruns, Th. "The influence of different vibration exciter systems on high frequency primary calibration of single-ended accelerometers", Metrologia, 47(1), pp. 58-64 (2010).

17. Bruns, Th., Link, A. and Täubner, A. "The influence of different vibration exciter systems on high frequency primary calibration of single-ended accelerometers: II", Metrologia, 49(1), pp. 27-31 (2012).

18. Shokrollahi, S. and Adel, F. "Effects of accelerometer mounting methods on quality of measured FRF's", International Congress on Sound and Vibration, ICSV21, Beijing, China, (2014). Accessed 3 January 2016:

http://www.iiav.org/arvhives_icsv_last/2014_icsv21/ content/papers/papers/Full_paper_655_ 20140423063601769.pdf.

19. Serridge, M. and Licht, T.R., Piezoelectric Accelerometers and Vibration Preamplifiers: Theory and Application Handbook, Bruel \& Kjaer, Denmark (1987).

20. Wagner, J. and Burgemeister, J. "Piezoelectric accelerometers: Theory and application", Metra Messund Frequenztechnik, Germany (2012). Accessed 19 June 2016: http://www.mmf.de/manual/ transducermane.pdf

21. Available from: www.globalsensortech.com/media/ CA-YD-1181.pdf.

\section{Appendix}

The ability to estimate the transmissibility permits to characterize the accelerometer input-output behavior in the time and frequency domains. There is no known criterion to quantify the parameters of the simulation in this case and explicit parameters are impossible to identify due to vast variation in mounting conditions and structures that may be subjected to testing. Hence, it cannot exactly indicate the transmissibility. However, one can determine the transmissibility of motion with some degrees of approximation.

The frequency response curve of accelerometer type CA-YD-1181 is shown in Figure A.1, in which the mounting resonance frequency, $f_{m}$, is about $40 \mathrm{kHz}$ for stud mounting $\left(\omega_{m}=2 \pi f_{m}\right)$. Wax mounting is stiffer than magnet mounting, but both of them are

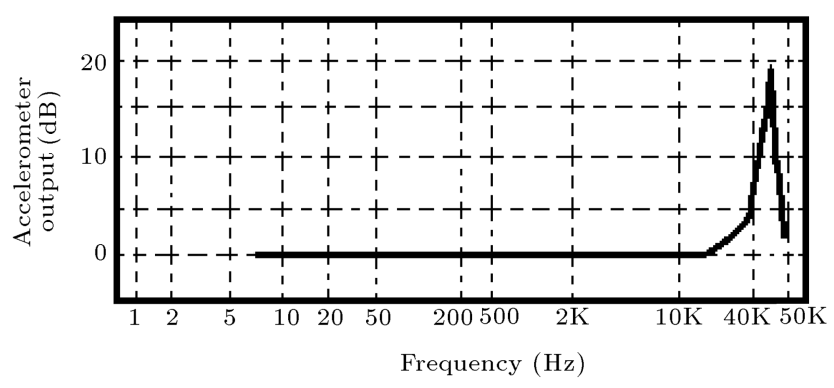

Figure A.1. Frequency response of SINOCERA accelerometer type CA-YD-1181 [21]. 




Figure A.2. Typical frequency response of an accelerometer mounted with a thin layer of wax [8].

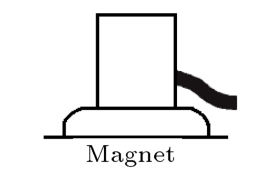

Maximum temperature: Up to $250^{\circ} \mathrm{C}$

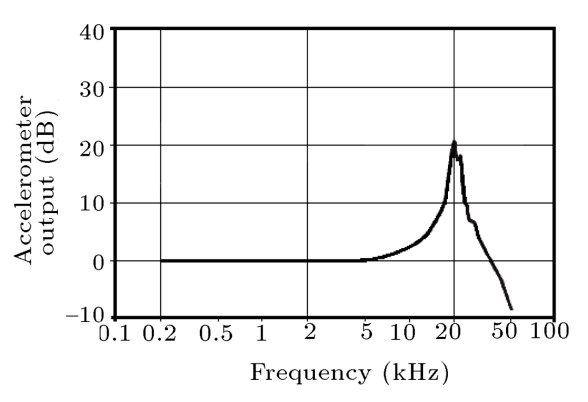

Figure A.3. Typical frequency response of a magnetically mounted accelerometer [8].

not as stiff as the stud mounting (see the comparisons in Figures 7 to 9 ). Thus, the mounted resonances in these two cases must be lower than that in a stud case; i.e. $\omega_{m}$ (magnet) $<\omega_{m}($ wax $)<\omega_{m}$ (stud). It is very useful to accurately determine the mounted resonance frequency of the accelerometer in wax and magnet conditions; but, at times, it is difficult in practice. Thus, we used the typical frequency response curves given in Figures A.2 and A.3 from ISO 5348 standard [8], i.e. we selected it approximately equal to $30 \mathrm{kHz}$ and $20 \mathrm{kHz}$ for wax and magnet conditions, respectively.

The resonance of the accelerometer alone will be most likely higher than that of mounted resonance frequency; therefore, we can assume the resonance of the accelerometer itself, $f_{s}$, to be about $60 \mathrm{kHz}$ $\left(\omega_{s}=2 \pi f_{s}\right)$.

The stiffness ratio, $r$, is a function of piezo-crystal stiffness, $k_{s}$, and mounting stiffness, $k_{m}$. Since $k_{s}$ is a constant quantity, the variation of stiffness ratio, $r$, could only be a function of $k_{m}$. Using the engineering judgment, three suitable values for stiffness ratio, $r$, are estimated. The connection stiffness in stud mounting is assumed to be equal to the stiffness of piezoelectric crystal. Thus, the value of $r$ for stud mounting is equal to 1 . Because magnet mounting was less reliable than wax mounting and both of them were less reliable than a stud, the values of 10 and 20 were selected for $r$ in wax and magnet mounting conditions, respectively. It should be pointed out that the quantifying process for parameter $r$ is arbitrary. It is derived from the main aim of the simulation, which is to find the transmissibility of mounting methods relative to each other. One might choose different values of stiffness ratio, $r$, for these three methods of mounting; but, these values must be increased from stud to magnet mounting methods anyway.

\section{Biographies}

Saeed Shokrollahi received his BSc degree in Mechanical Engineering from Shiraz University, Shiraz, Iran, in 1994; his MSc degree in Mechanical Engineering from Tehran University, Iran, in 1997; and his PhD degree in Mechanical Engineering from Amirkabir University of Technology, Tehran, Iran, in 2004. He is currently Assistant Professor of Mechanical Engineering in the Aerospace Engineering Department of Malek-Ashtar University of Technology, Tehran, Iran. His research interests are in the field of aeroelasticity, including the study of dynamic interaction between aerodynamic flow and elastic structures such as aircraft wings during high-speed flight. He has also undertaken research in experimental modal analysis, nonlinear identification, structural dynamics, and unsteady aerodynamics. He has supervised more than $30 \mathrm{MSc}$ theses and $3 \mathrm{PhD}$ dissertations since 2004.

Farhad Adel received his BSc degree in Mechanical Engineering from Sharif University of Technology, Iran, in 1998; and his MSc degree in Mechanical Engineering from Iran University of Science and Technology in 2001; and is currently studying for his $\mathrm{PhD}$ degree in Aerospace Engineering at Malek-Ashtar University of Technology, Tehran, Iran.

Hamid Ahmadian received his BSc degree from Iran University of Science and Technology in 1985, MSc degree from Amirkabir University of Technology in 1988, and PhD degree from Waterloo University in 1994, all in Mechanical Engineering. He is Professor of Vibration Engineering at Iran University of Science and Technology, Director of Center of Excellence in Experimental Solid Mechanics (CEEMech), and Editor-in-Chief of the journal "Theoretical and Applied Vibration and Acoustics". He held Visiting Professor Appointments at University of Swansea and University of Liverpool from 1994 to 2006. His current research interest is experimental vibration testing. 